\title{
A Worst Case Formula for a Communication and Computation Delay in NCS.
}

ROLF HENRY VARGAS VALDIVIA
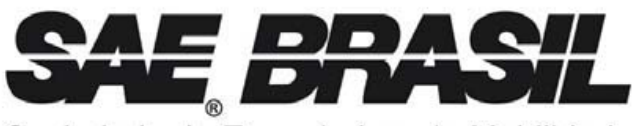

Sociedade de Engenheiros da Mobilidade

FILIADA À

SAEIIternational'

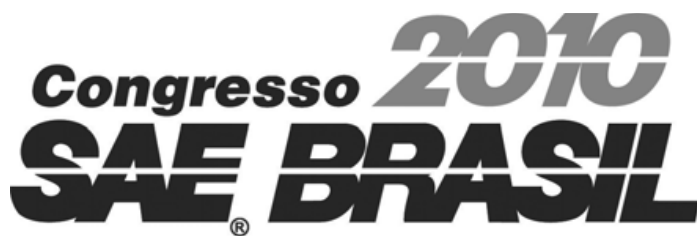

XIX Congresso e Exposição Internacionais de Tecnologia da Mobilidade

São Paulo, Brasil 05 a 07 de outubro de 2010

Este trabalho técnico/científico recebeu apoio financeiro do Conselho Nacional de Desenvolvimento Científico e Tecnológico - CNPq - Brasil

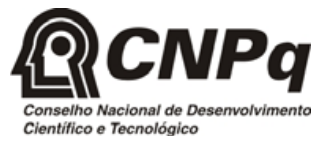

AV. PAULISTA, 2073 - HORSA II - CJ. 1003 - CEP 01311-940 - SÃO PAULO - SP 


\title{
A Worst Case Formula for a Communication and Computation Delay in NCS.
}

\author{
Rolf Henry Vargas Valdivia \\ Marcelo Lopes de Oliveira e Souza \\ National Institute of Space Research from Brazil
}

Copyright $(2010$ SAE International

\begin{abstract}
A major trend in modern aerospace and automotive systems is to integrate computing, communication and control into different levels of the vehicle and/or its supervision. A well fitted architecture adopted by this trend is the common bus network architecture. A Networked Control System (NCS) is called when the control loop is closed through a communication network. The presence of this communication network introduces new characteristics that must be considered at the design time of a control system. This work, still in development, focuses on a worst case formula for a communication (TDMA) plus computation (RMS) on a NCS. This formula, in a first instance, agrees with the simulated cases under the hypotheses and conditions when the NCS is composed by 1 actuator -1 sensor and when is composed by 2 actuators -2 sensors. In the future, we intent to generalize this formula and extend this study to NCS that uses other communication protocols or others computer schedulers.
\end{abstract}

\section{INTRODUCTION}

A real-time control system is a system in which the resulting performance depends not only on the correctness of the single control actions but also on the time at which the actions are produced [1]. Real-Time applications span a wide range of domains including industrial plants control, automotive, flight control systems, monitoring systems, multimedia systems, virtual reality, interactive games, consumer electronics, industrial automation, robotics, space missions, and telecommunications.

The operating system is the major architectural component responsible for ensuring a timely execution of all the tasks having some timing requirements. The objective of a real-time kernel is to ensure that each activity completes its execution within its deadline. A real-time task is said to be hard if missing a deadline may have catastrophic consequences on the controlled system, and is said to be soft if missing a deadline degrades the performance but does not jeopardize the correct system behavior.

Nowadays, the major trend in modern aerospace and automotive systems is to integrate computing, communication and control into different levels of the vehicle and/or its supervision. A well fitted architecture adopted by this trend is the common bus network architecture. A Networked Control System (NCS) is called when the control loop is closed through a communication network (Figure 1). 


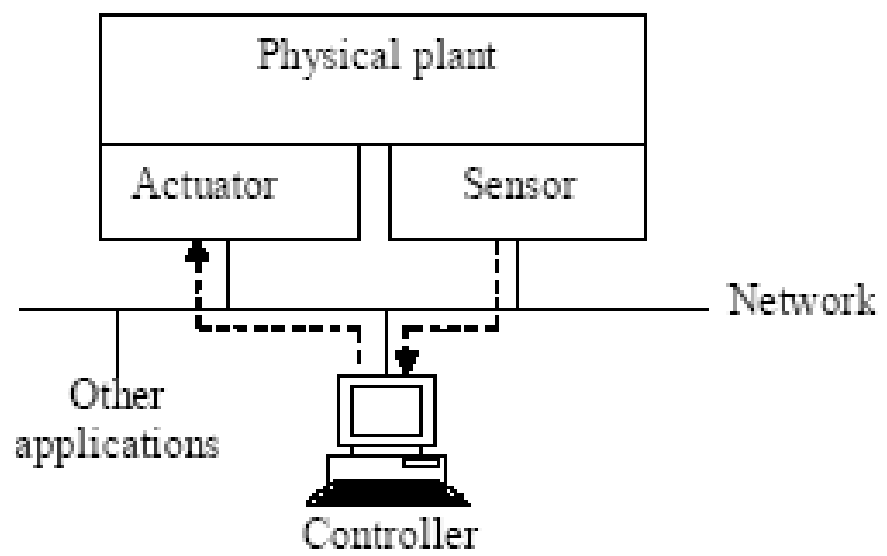

Figure 1. A general Networked Control System framework.

According [2], "the insertion of the network communication in the feedback control loop enables to improve, efficiency, flexibility, dependability and modularity of the systems, provides the features to easy installation and reconfiguration and can also reduce the setup and maintenance costs".

However, this has many impacts. The network implementation in the control systems makes the analysis and design of an NCS more complex. If this architecture is chosen for a real-time control system, the conventional control theories with many ideal assumptions, such as nondelayed calculations and transportation signals, must be re-evaluated before they can be applied to the NCSs.

The most important property of a real-time system (with or without network) is not high speed, but predictability. In a predictable system, we should be able to determine in advance whether all the computational activities can be completed within their timing constraints. To reach this predictability, this work, shows a formula for the worst case transmission delay in a NCS. This formula agrees with the simulated cases under certain hypotheses and conditions. The simulations were done with the help of the TrueTime toolbox and two configuration of NCS were tested: when the NCS is composed by 1 actuator -1 sensor and when the NCS is composed by 2 actuators -2 sensors.

\section{MODEL DESCRIPTION}

Our networked control system is composed by a control computer with Rate Monotonic Scheduler policy and, a communication network with TDMA access.

In this work, we have a particular interest on the Total delay $\left(\tau_{\text {Total }}\right)$ of a message in a NCS configuration. This Total delay is showed in Figure 2. We define the Total delay as the time interval since the start of one task in the sensor until its conclusion in the respective actuator of one control loop. This total delay must include the process time of the computer and the transmission time of the network, at minimum.

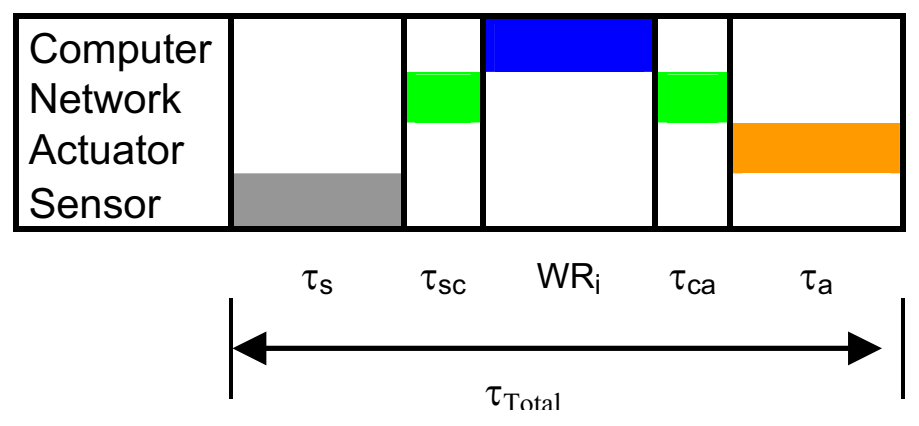

Figure 2. Sequence of tasks to be executed. 
The Figure 2. shows the sequence of the execution of tasks on the devices that composed a NCS. The times in Figure 2 are sensor time $\left(\tau_{\mathrm{s}}\right)$, transmission time from sensor to controller $\left(\tau_{\mathrm{sc}}\right)$, time to process the task " $i$ " assuming the worst case scenario $\left(\mathrm{WR}_{\mathrm{i}}\right)$, transmission time from controller to actuator $\left(\tau_{\mathrm{ca}}\right)$, the actuator time $\left(\tau_{\mathrm{a}}\right)$.

So, to reach high levels of predictability, the sequence and the priorities of the task must be considered. It's important to say that in some architectures identify the transmission times in the network could be more difficult than the execution time in the processor. Following, a brief description of the computer and communication schedulers employed in this work.

\section{RATE MOTONIC SCHEDULER}

We assume that in the computer is implemented a Rate Monotonic Scheduler (RMS) policy because is the most common priority-based method for scheduling a set of periodic tasks. It assigns to each task a priority directly proportional to its activation frequency, so that tasks with shorter period have higher priority. Since a period is usually kept constant for a task, the RMS algorithm implements a fixed-priority assignment in the sense that tasks priorities are decided at task creation and remain unchanged for the entire application run. RMS is typically preemptive, although it can also be used in a nonpreemptive mode. Liu and Layland [3] showed that RM Sis optimal among all static scheduling algorithms in the sense that if a task set is not schedulable by RMS, then the task set cannot be feasibly scheduled by any other fixed priority assignment.

WORST CASE RESPONSE TIME - The critical instant is the instant in which one task is triggered and all other taks with higher priority than it are triggered too. So the elapsed time since its trigger until its complete execution is called worst case response time (WR).

The expression to calculate the WR time is [4]:

$$
W R_{i}=C_{i}+\sum_{j \in h p(i)}\left\lceil\frac{W R_{i}}{T_{j}}\right\rceil \cdot C_{j}
$$

Where $\mathrm{WR}_{\mathrm{i}}$ is the worst case response time of the task " $\mathrm{i}$ ", $\mathrm{C}_{\mathrm{i}}$ is the computation time of the task " $\mathrm{i}$ ", $T$ is the period and the subindex "hp(i)" refers to the set of tasks with higher priority than " $i$ ". The ceiling function $\lceil\mathrm{x}\rceil$, returns the minimum integer greater than $\mathrm{x}$. It is important to say that Equation 1 is a recursive equation, so are needed iterative methods to solve it.

\section{NETWORK WITH TDMA ACCESS}

Consider a communication protocol with TDMA policy as medium access control. In protocols with TDMA access, each node is allowed to use all the bandwidth of the network by a fixed time interval called slot (S). The periodic sequence of slots to be transmitted is called TDMA round (TDMA $A_{\text {Round }}$ ). The Figure 3 shows the configuration of a TDMA Round. $_{\text {. }}$

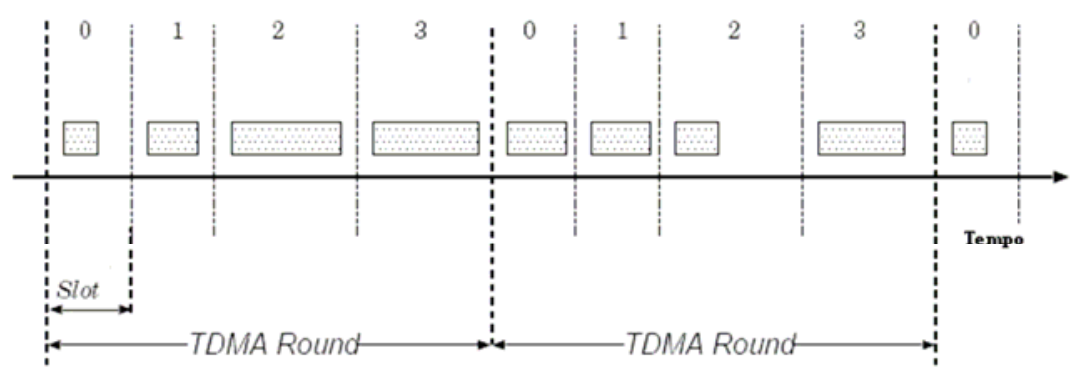

Figure 3. TDMA Round $_{\text {configuration. }}$ 
The Figure 3 shows little spaces between the slots. These spaces actually exist. The structure of one slot is composed by four phases: 1) Pre-Sending Phase (PSP); 2) Transmission Phase (TP); 3) Post-Receiving Processing Phase (PRP); 4) Idle. The only phase that uses the communication channel is the transmission phase. All the others involved intrinsic operations of the commmunication process (packet and unpacket the message, error checking, parity checking, etc). It is important to said that the duration of these phases depends directly on the hardware where it is implemented. In this work it is assumed that the slot size includes all the phases needed to transmission.

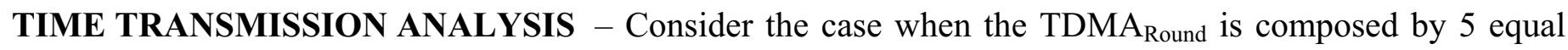
size, constants slots where every slot appeares once in a TDMA round as shows in Figure 4.

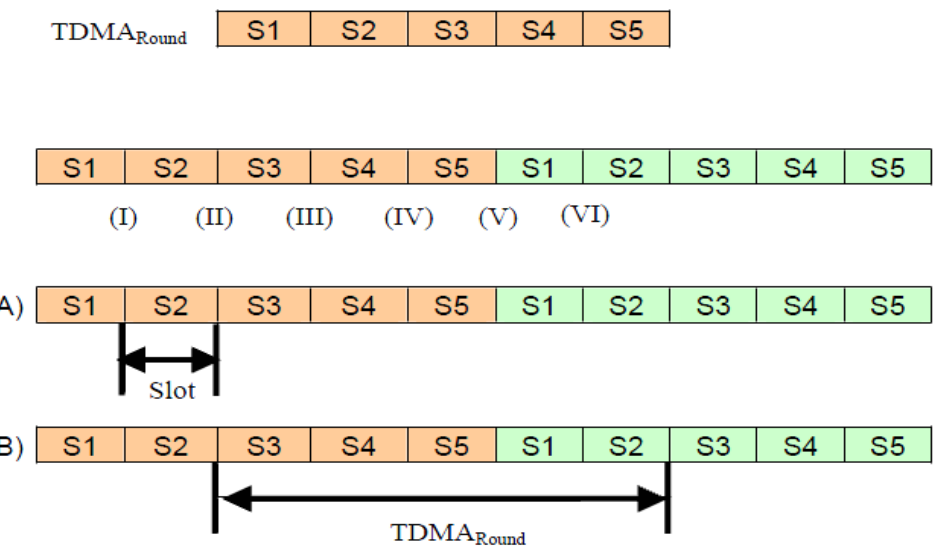

Figure 4. Transmission time analysis in TDMA network.

Lets suppose that the minimum time needed to do a transmission between two components is equal to one slot, it means, that in intervals of time smaller than one slot is not possible to transmit, so the component must wait until the next designated slot to transmit. Now, we are going to analize the transmission time of the points (I) to (VI). Suppose that the slot designated to transmit is the slot S2. If the transmission request coincides with the point (I) of the Figure 4a, so the elapsed time needed to do a transmission is just "one" slot. If the transmission request coincides with the point (II) of the Figure 4b, so the elapsed time needed to do the transmission is 5 slots.(it waits 4 slots and transmits only in the last). If the transmission request coincides with the point (III), so the elapsed time needed to do the transmission is 4 slots. If the transmission request coincides with the point (IV), so the elapsed time needed to do a transmission is 3 slots. If the transmission request coincides with the point $(\mathrm{V})$, so the elapsed time needed to do a transmission is 2 slots. If the transmission request coincides with the point (VI), so the elapsed time needed to do a transmission again is 1 slot.

Even the transmission request starts at the middle of any slot, this analisys shows that exists a minimum and maximum time to do a transmission in a network with TDMA access. The minimum time is represented by case $\mathrm{A}$ in Figure 4, when the transmission request coincides with the start of the slot S2, therefore the minimum time to transmit a message is one slot.

The maximum time is represented by case B in Figure 4, when the transmission request coincides with the end of the slots S2. So the maximum time to transmit a message is equal to one TDMA Round. This result will be usefull for the simulation cases.

The time analysis of a time-triggered system (computer, network, etc) is completely different from an eventtriggered system, it is enough to notice that it is not necessary that all nodes must transmit at a certain time to get a delay of one TDMA $A_{\text {Round. }}$ The one TDMA $A_{\text {Round }}$ delay could happened just with one node using the TDMA network. 
EQUATIONS INCLUDING COMPUTER AND NETWORK - Until here we see that exist activities in the computer (processing) and the network (transmission) that spend time. We also see that these devices are present in NCS.

Based on Figure 2, were done the following assumptions over the components of the NCS:

- The tasks in the sensors are time triggered and the execution time $\left(\tau_{\mathrm{s}}\right)$ is desconsidered.

- The control tasks in the computer are also time triggered.

- The tasks in the actuators are event triggered, it means, the actuator only executes a task is a new data arrives by the network.

- The actuator execution time $\left(\tau_{\mathrm{a}}\right)$ is desconsidered.

- Even the slot can change its size, it is assumed that constant size slot for all the transmission of the components.

- Exists an offset of one TDMA Round between the start of the tasks in the sensors and the start of the tasks in the computer.

- The TDMA $\mathrm{R}_{\text {Round }}$ is ordered in descendent priority, it means, let the set of " $n$ " tasks where the highest priority is the task " 1 " and the lowest the task " $n$ " (priority stablish by the periods in the computer). So the TDMA is formed in this way: $\mathrm{S}_{\mathrm{S} 1}-\mathrm{S}_{\mathrm{A} 1}-\ldots-\mathrm{S}_{\mathrm{Sn}}-\mathrm{S}_{\mathrm{An}}$. Where SS1 is the slot designated to the message transmission of the sensor " 1 " to computer and inmediately after SA1 the slot designated to the transmission of the computer to the actuator " 1 ".

- All the sensors start to transmit at $\mathrm{t}=0$.

Having all these considerations in mind, it is possible to formulated an equation for the worst case transmission plus computation delay (WT). According to [5], the worst case transmission plus computation delay is:

$$
W T_{i}=\left\lceil\frac{W R_{i}}{S}\right\rceil \cdot S+2 \cdot T D M A_{\text {Round }}
$$

Where " $\mathrm{S}$ " represents the slot size in time units and $\mathrm{WR}_{\mathrm{i}}$ is the worst case response time of the task "i". The right side of Equation 2 is related with the network, as explained before, the worst delay that a message

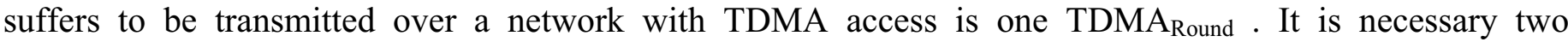
transmission so thats why we have it duplicated.

The left side of Equation 2 is related with the worst case response time of one task $\left(\mathrm{WR}_{\mathrm{i}}\right)$ in the computer. How WRi could be a real posivitve number, so the ceiling function converts it to a intger quantity of slots. Note that the WT delay could be greater than TDMA Round.

\section{TRUETIME}

It is a simulator based on Matlab/Simulink for control systems in real-time. With TrueTime is possible simulate the timing behavior of real-time multi-task kernels and study the effects of the CPU and network "schedulings" on the performance of the control system. Several scheduling policies can be defined, and control tasks can be implemented using $\mathrm{C}$ functions, $\mathrm{M}$ files, or by blocks diagrams of the Simulink [6]. The Figure 5 presents the libraries of the TrueTime in the form of blocks of Simulink. 


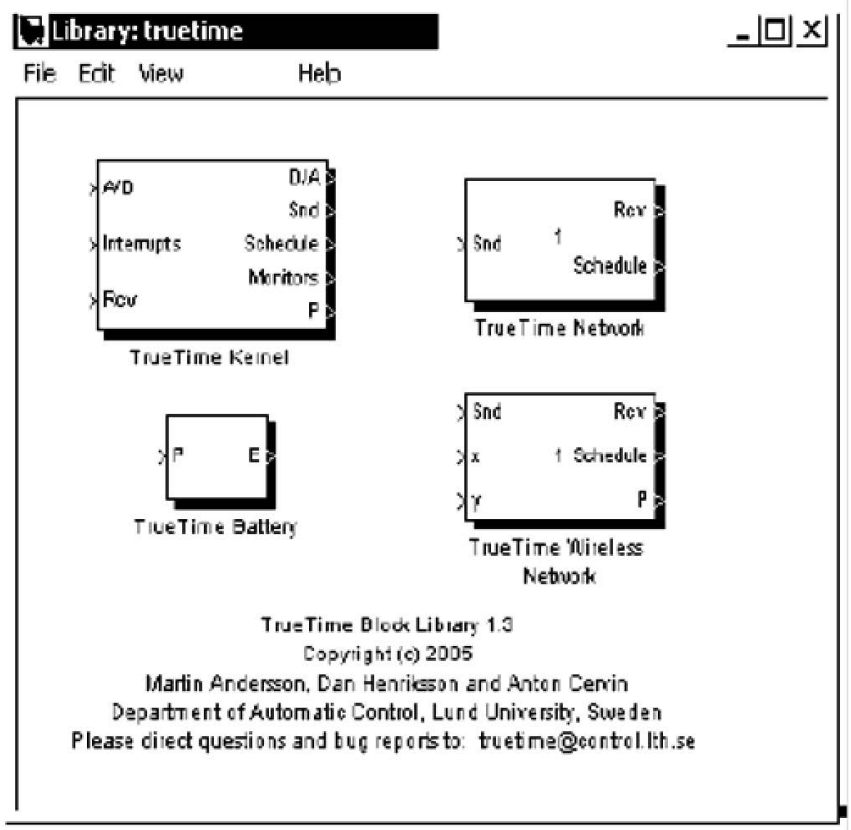

Figure 5. Simulink TrueTime blocks libraries.

Six simple models of wired networks can be supported: CSMA/CD (e.g. Ethernet), CSMA/AMP (e.g. CAN), Round Robin (e.g. Token Bus); FDMA, TDMA (e.g. TTP), and Switched Ethernet (e.g. AFDX). Additionaly, 2 models of wireless networks are supported, the WLAN and Zigbee.

This toolbox helps us in all the simulation cases done in this work.

\section{SIMULATIONS}

To reach our objectives, it is considered a continuous general second order systems as a plant and the control signal is calculated by a digital proportional controller. We choose a simple plant and control models, because we want to identify the effects of the total delay and determine its impact on the performance of the system.

Three study cases are presented. The first one represents the situation of one control tasks over an architecture with network (Case 1). The second case represents two control tasks with the same time period and with a network (Case 2a). The third case, represents also two control task but with different time period with network (Case 2b).

CASE 1: 1 sensor -1 actuator, to illustrate this case we choose a system with one task with $\mathrm{C}_{1}=5 \mathrm{~ms} ; \mathrm{T}_{1}=10$ ms. The Figure 6 shows the network and computer schedulers for this case. In Figure 6, the two first curves (solid) represents the activity of the network, the first curve shows the activity of the sensor (sensor to computer transmission) and the second the activity of the computer (computer to actuator transmission); and the third line (dashed) represent the activity of the control task in the computer. In both cases the high level represents an action (transmitting or processing, respectively) and the low level a no task activity, there is a mid-level (not showed), it represents the waitting for transmit a message or process a task, respectively.

By Equation 2, the worst transmission delay is $9 \mathrm{~ms}$. Assuming that 1 slot is equal to $1 \mathrm{~ms}$. In this case the worst transmission delay never happened, the maximum delay is $8 \mathrm{~ms}$. 


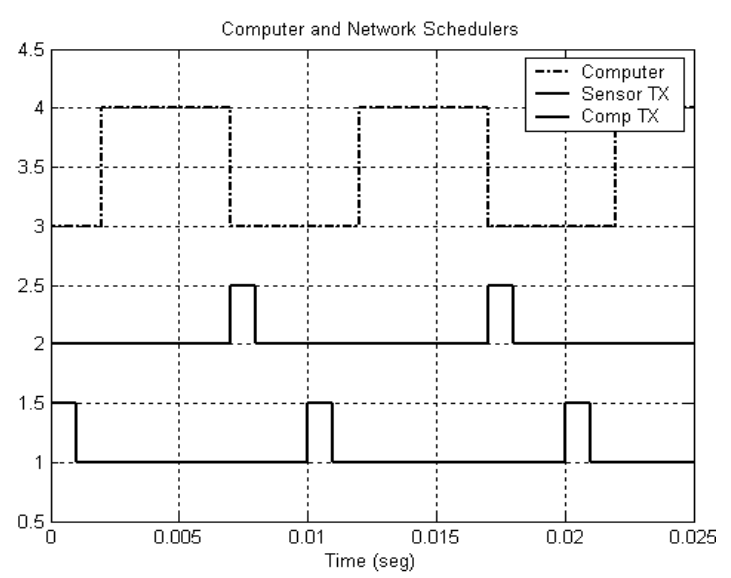

Figure 6. Computer plus network schedulers for case 1

CASE 2a: 2 sensors - 2 actuators with same time period, to illustrate this case we choose a system with two tasks with $\mathrm{C}_{1}=5 \mathrm{~ms} ; \mathrm{T}_{1}=15 \mathrm{~ms}$ and $\mathrm{C}_{2}=7 \mathrm{~ms} ; \mathrm{T}_{2}=15 \mathrm{~ms}$. The Figure 7 shows the schedulers for this case. This values represents a schedulable system.

Considering the numerical example data and that we have a constant slot size equal to $1 \mathrm{~ms}$, so the $\mathrm{TDMA}_{\text {Round }}$ value is $4 \mathrm{~ms}$. By Equation 2, the worst transmission delay for each tasks are $13 \mathrm{~ms}$ and $20 \mathrm{~ms}$.

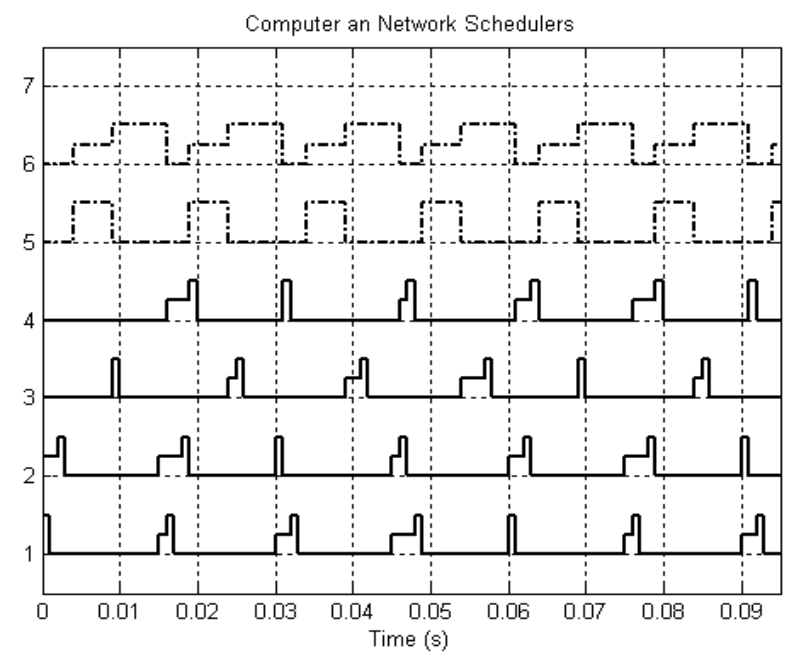

Figure 7. Computer plus network schedulers for case 2a.

Table 1: Delay values of tasks of Figure 6.

\begin{tabular}{|c|c|c|}
\hline & $\begin{array}{c}\text { Task 1 delay } \\
(\mathrm{ms})\end{array}$ & $\begin{array}{c}\text { Task 2 delay } \\
(\mathrm{ms})\end{array}$ \\
\hline 1$)$ & 10 & 20 \\
\hline 2$)$ & 11 & 17 \\
\hline 3$)$ & 12 & 18 \\
\hline 4$)$ & 13 & 19 \\
\hline
\end{tabular}

The table 1 shows the delay sequence that repeats all the time. As we see, the maximum values agrees with the value of equation 2 .

CASE 2b: 2 sensors -2 actuators with different time period, now to illustrate this case we choose a system with two tasks, $\mathrm{C}_{1}=3 \mathrm{~ms} ; \mathrm{T}_{1}=7 \mathrm{~ms}$ and $\mathrm{C}_{2}=9 \mathrm{~ms} ; \mathrm{T}_{2}=19 \mathrm{~ms}$. The Figure 8 shows the schedulers for this case. 
The Figure 8 shows a part of the scheduler of this case. In this part the worst delay for the second task is present: Notice that this worst delay not appearance at the beginning. The delay sequence for the first task, 10-11-08- 09 msec and the delay sequence of the second task is showed in the Table 2. Once more the maximum value agrees with the value of Equation 2.

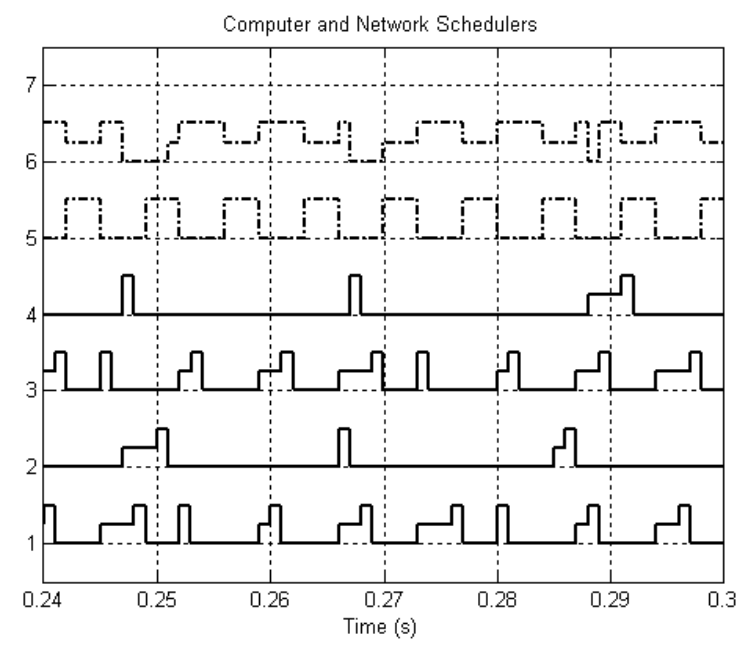

Figure 8. Computer and network schedulers for case $2 \mathrm{~b}$.

Table 2. Delay values of second taskfor case $2 b$.

\begin{tabular}{|l|c|c|c|c|c|}
\hline & $\begin{array}{c}\text { Task 2 } \\
\text { Delay } \\
(\mathrm{ms})\end{array}$ & $\begin{array}{c}\text { Task 2 } \\
\text { Delay } \\
(\mathrm{ms})\end{array}$ & $\begin{array}{c}\text { Task 2 } \\
\text { Delay } \\
(\mathrm{ms})\end{array}$ \\
\hline 1$)$ & 24 & $11)$ & 22 & $21)$ & 24 \\
\hline 2$)$ & 21 & $12)$ & 23 & $22)$ & 25 \\
\hline 3$)$ & 22 & $13)$ & 20 & $23)$ & 22 \\
\hline 4$)$ & 23 & $14)$ & 21 & $24)$ & 23 \\
\hline 5$)$ & 20 & $15)$ & 26 & $25)$ & 24 \\
\hline 6$)$ & 21 & $16)$ & 23 & $26)$ & 21 \\
\hline 7$)$ & 22 & $17)$ & 20 & $27)$ & 22 \\
\hline 8$)$ & 23 & $18)$ & 25 & $28)$ & 23 \\
\hline 9$)$ & 20 & $19)$ & 22 & $29)$ & 24 \\
\hline 10$)$ & 21 & $20)$ & 23 & & \\
\hline
\end{tabular}

\section{CONCLUSIONS}

In this work, a worst case formula for communication and computation delay in NCS is showed.

The worst case response time of a task in the computer and in the network were analyzed independly.

We choose a RM scheduler and TDMA protocol by its simplicity and determinism but when we use both in an integrated design the result could be not well comported as we expected. The presence of a worst delay case is only determined with an extensive analysis of the delay until the delay sequence be discovered.

The advantage to use the TDMA is that even the delay varies, it is a priori known and techniques to compensate it can be used. The theoretical results are not general enough to represent any NCS. The total delay must be reanalyzed under different assumptions and configurations.

We pretend to use this delay analysis in future designs of satellite control of attitude and orbit (SCAO) systems. 


\section{CONTACT INFORMATION}

For more information and/or any questions or doubts, please contact the authors through e-mails, rolf.vargas@gmail.com; marcelo@dem.inpe.br.

\section{ACKNOWLEDGMENTS}

The first author thanks CAPES by supporting him during this research; the second author thanks INPE/DMC by supporting him and the Laboratory of Computer Environments for Simulation, Identification, and Modeling - LABSIM2 of AOCS where this work is being done.

\section{REFERENCES}

1. J. Stankovic, "Misconceptions About Real-Time Computing: A Serious Problem for Next-Generation Systems", IEEE Computer,Vol. 21, No. 10, pp. 10- 19, October 1988.

2. R. Raji, "Smart network for control", IEEE Spectrum, Vol. 31, No. 6, pp 49-55, 1994.

3. C. L. Liu, James W. Layland; "Scheduling Algorithms for Multiprogramming in a Hard-Real-Time Environment" Journal of the ACM (JACM), Vol. 20, Issue 1, January 1973.

4. M. Joseph, P. Pandya, Finding response times in a real-time system. The Computer Journal, v. 29, n. 5, p. 390-395, 1986.

5. R.H.V. Valdivia, Influência dos agendadores da computação (RMS) e da comunicação (TDMA) na estabilidade de um sistema de controle por rede. PhD Thesis INPE-15692-TDI/144, 2009.

6. A. Cervin, D. Henriksson, B. Lincoln, J. Eker, K. Årzén: "How Does Control Timing Affect Performance?". IEEE Control Systems Magazine, 23:3, pp. 16--30, June 2003. 
The Engineering Meetings Board has approved this paper for publication. It has successfully completed SAE's peer review process under the supervision of the session organizer. This process requires a minimum of three (3) reviews by industry experts.

ISSN 0148-7191
Positions and opinions advanced in this paper are those of the author(s) and not necessarily those of SAE. The author is solely responsible for the content of the paper.

SAE Customer Service:

Tel: 877-606-7323 (inside USA and Canada)

Tel: 724-776-4970 (outside USA)

Fax: 724-776-0790

Email: CustomerService@sae.org

SAE Web Address: http://www.sae.org

Printed in USA 